\title{
COLLECTIVE EFFECTS OF SUPERNOVA EXPLOSIONS IN A STARBURST NUCLEUS
}

\author{
Kohji Tomisaka and Satoru Ikeuchi \\ Tokyo Astronomical Observatory, University of Tokyo, \\ Mitaka, Tokyo 181, Japan
}

\begin{abstract}
The collective effects of supernova ( $\mathrm{SN}$ ) explosions in the nucleus of a starburst galaxy are studied. It is shown that a large wind bubble with the size of a few hundred parsecs in the nucleus of a starburst galaxy will expand for SN explosion rates of 0.1 per year or greater. The bubble gradually elongates due to density stratification. Finally, the shell breaks near the top and the hot matter flows through the cylindrical shell up to 1-2 $\mathrm{kpc}$ above the disk plane. We will discuss the X-ray emission in the halos of galaxies such as M82 and NGC 253 and the distribution of molecular gas in such galaxies in relation to the starburst phenomenon.
\end{abstract}

Introduction: There has been increasing interest in star formation bursts in many galaxies. We summarize here the observational results of well-known examples of starburst nuclei in the nearby galaxies M82 and NGC 253.

Disk Component: The disk component of a starburst nucleus is observed as a disk or a torus with radius $\sim 200 p c$ in a map at near and far IR bands. Recently, very young supernova remnants (SNRs) were found in M82 (Kronberg et al. 1985) and NGC 253 (Turner and Ho 1985). These objects trace the IR disk. CO observations, which give us an overall distribution of molecular matter, have shown that $\mathrm{H}_{2}$ gas forms a "200-pc ring" and a $\mathrm{CO}$ ridge or spur-like structure extends perpendicularly from the galactic plane towards the halo (Olofsson and Rydbeck 1984, Nakai et al. 1987, Lo et al. 1987). The $\mathrm{CO}$ ridge surrounds both the IR disk and the radio SNRs cylindrically up to $500 \mathrm{pc}$ from the plane of the disk.

Extended Component: As well as the CO ridge, an extended component is seen in X-ray emission, extending perpendicularly to the disk up to $2-3 \mathrm{kpc}$ in M82 (Watson et al. 1984, Kronberg et al. 1985) and $1.2 \mathrm{kpc}$ in NGC 253 (Fabbiano and Trinchieri 1984). The extended X-ray emission strongly suggests that the upper halo region is shock-heated by a burst of SN explosions in the disk region. The spur-like structure seems to consist of a gas which resided in the disk and was pushed aside by the X-ray emitting hot gas, as proposed by Unger et al. (1984). On the outflow around the starburst nucleus, Chevalier and Clegg (1985) have analytically studied the spherically symmetric wind. In the present paper, by full two-dimensional numerical hydrodynamics we study the evolution of wind bubbles formed by frequent $\mathrm{SN}$ explosions after the starburst in the nuclear regions of galaxies.

Model: We assume that star formation and a subsequent SN burst begin abruptly in the interstellar medium which was originally settled in a static equilibrium around a nucleus. Initial Condition: We assume that the gravitational field is dominated by the stellar component whose density obeys King's distribution. The gravitational force is balanced by the centrifugal force and the pressure gradient. Here, we assume that the rotation is uniform in the $z$-direction and on the disk plane the centrifugal force is balanced by a part $\left(e^{2} ; e=0.9\right)$ of the gravitational force. To fit the rotation curve along the major axis of M82, the parameters are determined as follows: King's core radius $r_{c} \approx 350 p c$, peak rotation velocity $v_{\phi m a x} \approx 60 \mathrm{kms}^{-1}$, and central stellar density $\rho_{c} \approx 1.4 \times 10^{-22}\left(v_{\phi \max } / 60 \mathrm{~km} \mathrm{~s}^{-1}\right)^{2}\left(r_{c} / 350 \mathrm{pc}\right)^{-2} e^{-2} \mathrm{~g} \mathrm{~cm}^{-3}$. Further, with the assumption that the gas is entirely isothermal, we take the three-dimensional random velocity of clouds as the isothermal sound speed $c_{s}=30 \mathrm{~km} \mathrm{~s}^{-1}$ (Olofsson and Rydbeck 1984). 
SN Explosions: From the distribution of compact nonthermal radio sources, we suppose that the SN burst occurs homogeneously within a disk of diameter $d \approx 300 p c$ and thickness $h \approx 50 p c$. We simulate the $S N$ burst by continuous deposition of energy $E_{S N} \times r_{S N}$ and mass $M_{e j} \times r_{S N}$ into the above disk, where $E_{S N}$ is the energy ejected by a $S N, M_{e j}$ is the mass ejected by a $S N$, and $r_{S N}$ is the $S N$ rate.

Results: We calculated six cases as summarized in Table 1. We will consider only two cases in the present paper due to space restrictions (see Tomisaka and Ikeuchi 1987 for complete results). Parameters in this simulation are $n_{0}$ (density at the center of the disk), $r_{S N}$, and $M_{e j}$. The explosion energy is fixed as $E_{S N}=10^{51} \mathrm{erg}$.

Case A: In Fig.1, we illustrate the time evolution of the gas flow. At stage (a) $(t=$ $\left.10^{6} \mathrm{yr}\right)$, the cooled dense shell $\left(n>30 \mathrm{~cm}^{-3}\right.$ and $T<10^{4} \mathrm{~K}$ ) is formed almost spherically, within which a hot rarefied cavity Table 1. The adopted parameters and the calculated X-ray luminosity.

\begin{tabular}{l|l:l|l:l|l}
\hline$n_{0}$ & $\begin{array}{l}r_{\mathrm{SN}} \\
\left(\mathrm{cm}^{-3}\right)\end{array}$ & $\begin{array}{l}M_{\mathrm{ej}} \\
\left(\mathrm{yr}^{-1}\right)\end{array}$ & $\begin{array}{l}L_{\mathrm{HRI}} \\
\left(M_{\odot}\right)\end{array}$ & $\begin{array}{l}\text { Age } \\
(\mathrm{Myr})\end{array}$ \\
\hline $\mathrm{A}$ & 20 & 0.1 & 30 & $210^{40}$ & 6 \\
$\mathrm{~B}$ & 20 & 0.1 & 10 & $210^{40}$ & 4 \\
$\mathrm{C}$ & 100 & 0.1 & 30 & $310^{40}$ & 10 \\
$\mathrm{D}$ & 20 & 0.01 & 30 & $110^{39}$ & 9 \\
$\mathrm{E}$ & 20 & 0.005 & 30 & $410^{39}$ & 11 \\
$\mathrm{~F}$ & 100 & 0.02 & 30 & $610^{39}$ & 9 \\
\hline
\end{tabular}
$\left(T \geq 3 \times 10^{7} K\right)$ spreads.

The bubble gradually begins to elongate in the $z$-direction as seen in Fig. $1 \mathrm{~b}\left(t=2 \times 10^{6} \mathrm{yr}\right.$ ). In Fig. 1c $\left(t=4 \times 10^{6} \mathrm{yr}\right)$, we can see that the outer shock front expands to $\sim 1 \mathrm{kpc}$ in the $z$-direction. Gas flowing out from the energy injection region soon becomes isotropic, and turns its direction upward at the inward-facing shock. As Rózyszka and TenorioTagle (1985) have pointed out, a part of the shell of the bubble which expands into the region with the large density gradient and is accelerated $(\partial \ln z / \partial \ln t<1)$ breaks due to a Rayleigh-Taylor instability. The instability grows near the $z$-axis and at age $t \approx 4 \times 10^{6} \mathrm{yr}$ the shell is broken. Fig. 1d shows the structure at $t=6.3 \times 10^{6} \mathrm{yr}$. It is found that a cylindrical wall with a radius $r \approx 600-700 p c$ is formed below $z \lesssim 1.6 \mathrm{kpc}$, through which hot gas is flowing upwards. Above $z \gtrsim 1.6 \mathrm{kpc}$ the dense wall is not formed and the hot gas pushes directly on the ambient matter. Such cylindrical walls with upward flowing hot gas, resembling chimneys, are also seen in superbubbles from sequential SN explosions in $\mathrm{OB}$ associations (Tomisaka and Ikeuchi 1986).

Effect of Supernova Rate: We can see the typical case of a low SN rate in Case E where

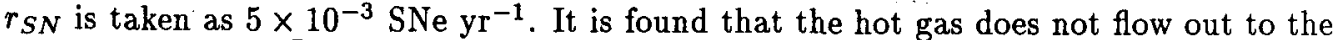
halo $z \sim 1 k p c$ in $10^{7} y r$. We show the structure at an age of $t=10^{7} y r$ in Fig. 2. Although the bubble elongates a little in the $z$-direction, the dense shell entirely surrounds the hot cavity and the hot gas is completely confined within the wall. In the parameter space of $\left(n_{0}, r_{S N}\right)$, the region where the hot gas halo is formed in $t \sim 10^{7} y r$ is shown in Fig. 3 . For both Cases D and F, the liberated energy of SNe is efficiently radiated away from the dense wall and the hot gas within the cavity, hindered by the wall, cannot escape to the halo.

Discussion: Fig. 4 shows the relation between the expected distribution of column density of neutral gas and that of soft X-ray intensity in the Einstein HRI band for the situation depicted in Fig. 1d. The ridge of neutral gas is located just outside the Xray emitting region. This agrees with the relative distribution in $\mathrm{M} 82$ of $\mathrm{H}_{2}$ (derived by Nakai et al. 1987) and of X-ray (Watson et al. 1984). These show that the observed gas distribution, in which the hot gas is surrounded cylindrically by the cooled gas, is well explained by this model. The starburst nucleus forms a galactic-scale bipolar flow in consequence of the active $\mathrm{SN}$ explosions. In Table 1, the X-ray luminosity obtained by the simulation is summarized. It is shown that the luminosity is $\sim 2 \times 10^{40} \mathrm{erg} \mathrm{s} \mathrm{s}^{-1}$ in the HRI band in Cases A-C, but in Cases D-F the luminosity is only $\sim 10^{39} \mathrm{erg} \mathrm{s} \mathrm{s}^{-1}$. 
This means that a SN rate higher than $\sim 0.1 y r^{-1}$ or a mechanical luminosity more than $3 \times 10^{42} \mathrm{erg} \mathrm{s}^{-1}$ is necessary to explain the observed X-ray luminosity. In Fig.5 we illustrate the profile of the X-ray intensity along the $z$-axis for Cases A and C. We also plot the observed intensity in M82 and NGC 253. Observed $I_{H R I}$ decreases from $z=500 p c$ to $z=2 k p c$ by only $1 / 10$; this fact fits well with our model calculation, although the expected surface brightness is smaller than that observed.

This work was supported in part by a Grant-in-Aid for Scientific Research from the Ministry of Education, Science, and Culture (61790072) in fiscal 1986 and 1987.

\section{References}

Chevalier, R. \& Clegg, A. W. 1985, Nature, 317, 44. Fabbiano, G. \& Trinchieri, G. 1984, Ap.J., 286, 497.

Kronberg, P. P., Bierman, P. \& Schwab, F. R. 1985, Ap.J., 291, 693.

Lo, K. Y. et al. 1987, Ap.J., 312, 574.

Nakai N. et al. 1987 submitted to Pub. Astr. Soc. Japan.

Olofsson, H. \& Rydbeck, G. 1984, Astr. Ap., 136, 17.

Rózyszka, M. \& Tenorio-Tagle, G. 1985, Astr. Ap., 147, 209.

Tomisaka, K. \& Ikeuchi, S. 1986, Pub. Astr. Soc. Japan, 38, 697.

Tomisaka, K. \& Ikeuchi, S. 1987, submitted to Ap.J.

Turner, J. L. \& Ho, P. T. P. 1985, Ap.J.(Letters), 299, L77.

Unger, S. W. et al. 1984 M.N.R.A.S., 211, 783.

Watson, M. G., Stanger, V. \& Griffiths, R. E. 1984, Ap.J., 286, 144.

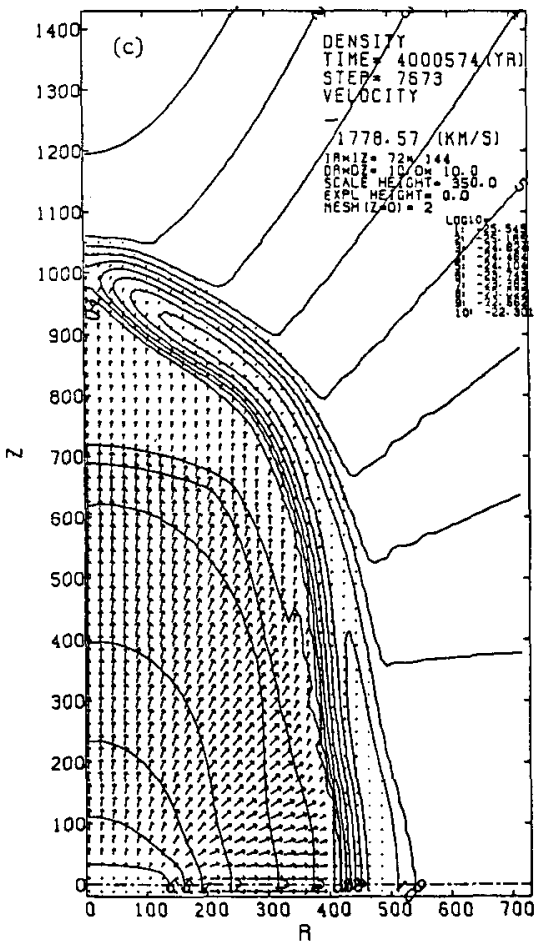

Fig.1: The evolution of the flow in Case $A$ (c) $t=4 \times 10^{6} \mathrm{yr}$, and (d) $t=6 \times 10^{6} \mathrm{yr}$ are illustrated.
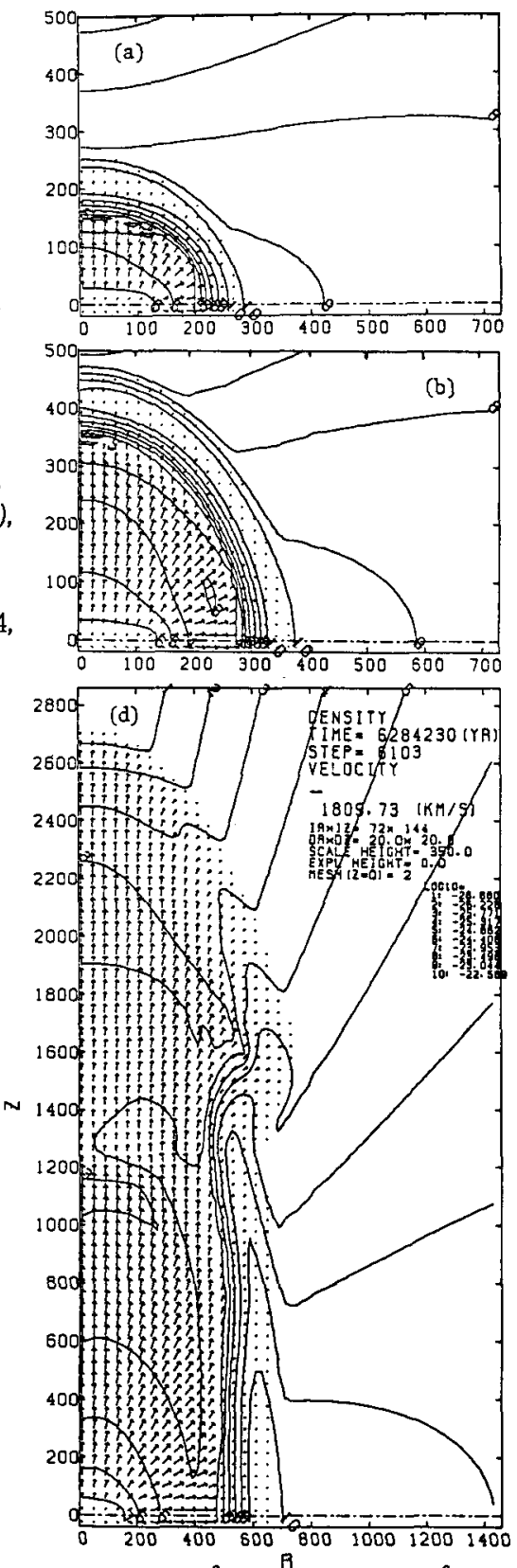

(a) $t=10^{6} \mathrm{yr}^{\mathrm{R}}$ (b) $t=2 \times 10^{6} \mathrm{yr}$ 

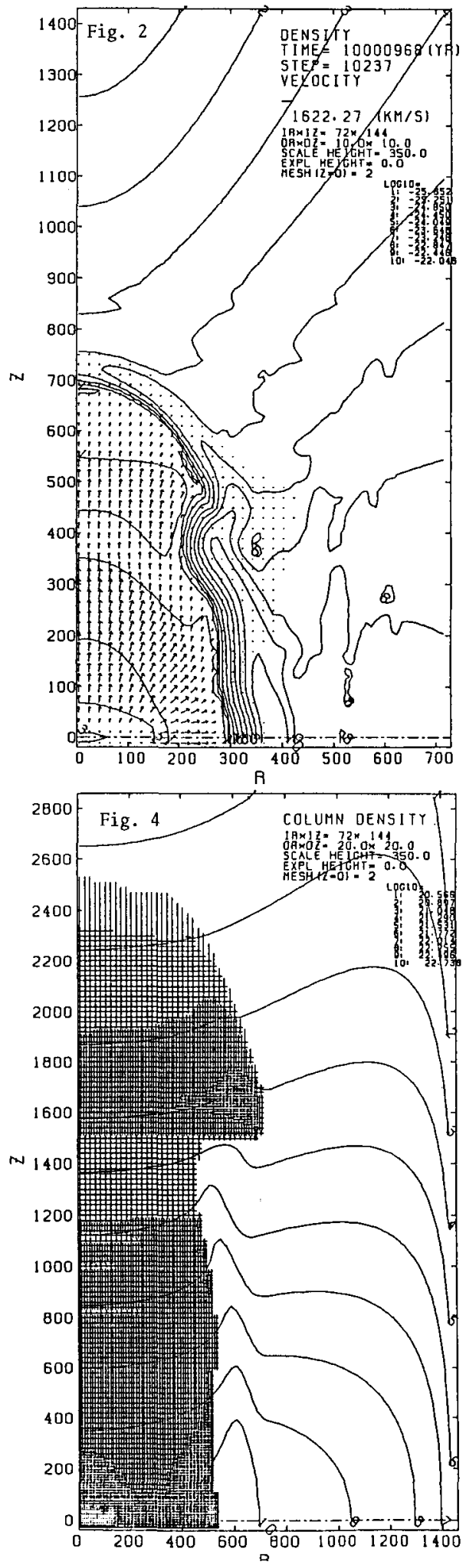
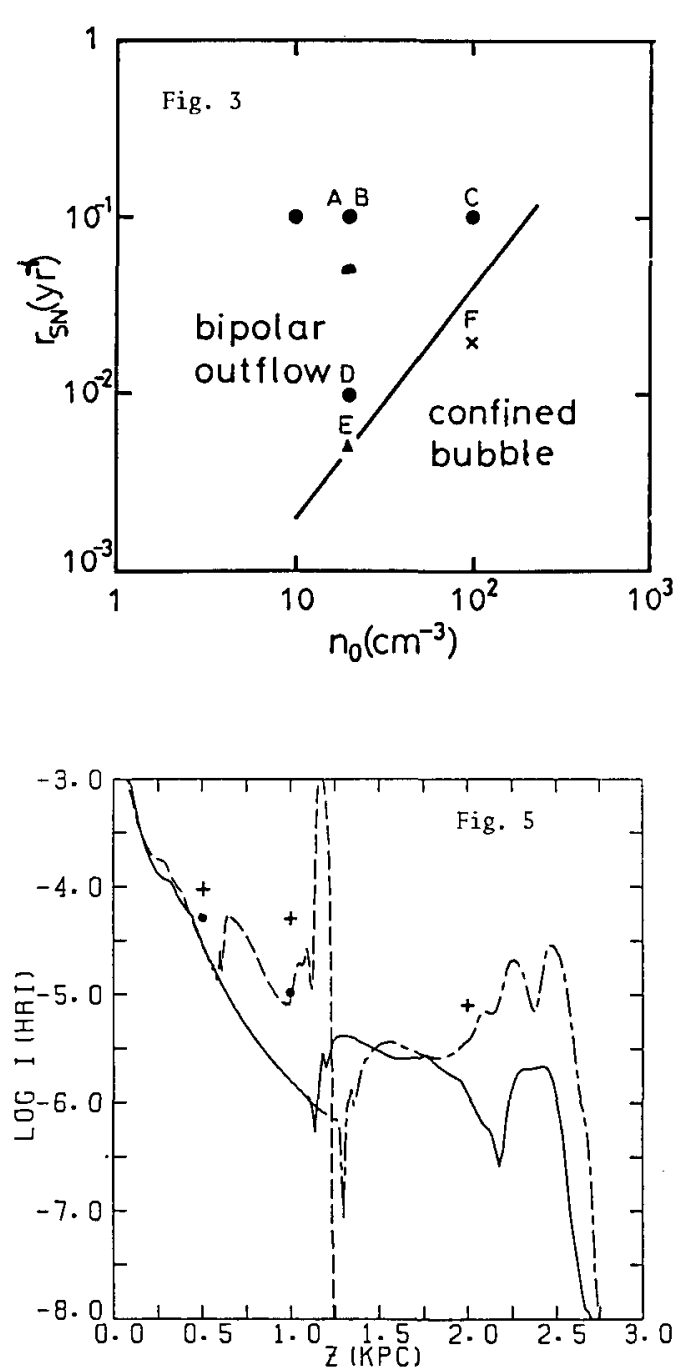

Fig.2: The same as in Fig.1 but for Case $\mathrm{E}$ at $t=10^{7} \mathrm{yr}$. It is clearly seen that in contrast with Case A the bubble is confined to the disk and does not deform much.

Fig.3: The parameter region where the hot gas halo extended to $z \approx 1 \mathrm{kpc}$ is formed in $t=10^{7} \mathrm{yr}$.

Fig.4: The column density of neutral gas and the soft $\mathrm{X}$-ray intensity for the case shown in Fig.ld.

Fig.5: The profile of $\mathrm{X}$-ray intensity in HRI band along the $z$ axis for Cases A(solid line at $t=6 \times 10^{6} \mathrm{yr}$ ), and $\mathrm{C}$ (dashed line at $t=7.5 \times 10^{6} \mathrm{yr}$, and dash-dotted line at $t=10^{7} \mathrm{yr}$ ). We also plot the observational data of M82(cross) and NGC 253 (circle). 